\title{
An End-Effector Based Upper-Limb Rehabilitation Robot: Preliminary Mechanism Design
}

\author{
Yekaterina Ponomarenko, Bauyrzhan Aubakir, Shahid Hussain and Almas Shintemirov \\ Department of Robotics and Mechatronics \\ Nazarbayev University \\ Astana, Kazakhstan, 010000 \\ Emails: shahid.hussain@nu.edu.kz, ashintemirov@nu.edu.kz
}

\begin{abstract}
Loss of upper limb function often appears after stroke. In this paper the 3D mechanism design for an electromagnetically powered end-effector based robotic manipulator was developed for post-stroke physical therapy. The proposed system delivers a dexterous performance and has a novelty among end-effector based rehabilitation robots. Its design incorporates curved links, which expand the manipulators performance abilities, and, as a result, increase the range of types of rehabilitation therapies. The proposed robotic manipulator can provide a patient with three and two degrees of freedom in shoulder and elbow joints, respectively. It has seven degrees of freedom, and is capable of $7 \mathrm{~kg}$ payload. The design of manipulators linkages was optimized to decrease the weight of the system.

Index Terms-Rehabilitation, upper limb, robots, stroke
\end{abstract}

\section{INTRODUCTION}

Neurological impairment after stroke frequently leads to the loss of the motor skills in the limb that affects the patients ability to perform activities of daily life. Training and rehabilitation therapy offer an opportunity for motor recovery [1]-[3].

Studies show that human brain is adaptable to changing demands and capable of self-reorganization. Training with specific exercises is necessary to induce nervous systems changes, provoke motor plasticity and, as a result, improve motor recovery [4]. Thus, to restore motor capabilities, the affected limb has to be exercised [5].

A therapy regime must be intensive, repetitive and of long duration. Conventional rehabilitation therapies are effort intensive. Physiotherapists have to assist manually the patient to conduct the exercises [6]. Training is exhausting for the therapist and, as a result, time limited [7]. This fact stimulates new approaches in rehabilitation in a way it becomes more effective and prolonged.

Robot-aided therapy is an emerging part of post-stroke rehabilitation care [8], [9]. Robotic exercisers provide intensive motor therapy, which can be performed in a repetitive, accurate and controllable manner [10], [11]. Moreover, the therapeutic action of the robot can be adjusted to the patients motor abilities [12], [13]. Training with mechatronic systems has mostly shown encouraging outcomes of rehabilitation [5], [14], [15]. Robotic devices may offer the required amount of motor practice and reduce the effort of therapists conducting the rehabilitation procedure [16], [17]. This paper focuses on the upper limb rehabilitation.
Several rehabilitation robots for the upper limb have been proposed. In general, there are two types of designs: exoskeleton [18]-[20] and robotic end-effector based [21] systems. In end-effector based robotic exercisers a patients hand is attached to a hand-holder having trajectories that simulate natural movements of the arm [16]. Examples of the endeffector based robots include MIT Manus [22], [23], ARM Guide [24], MIME [21].

The aim of this project was to design an end-effector based robot for upper extremity rehabilitation. The paper is organized as follows: Section II presents the design requirements, the concept and the mechanical model of the rehabilitation system. Section III illustrates and discussed the results, while Section IV comments on conclusion.

\section{Methodology}

\section{A. Proposed System Requirements}

A robotic device is required to provide exercise to the patients arm in the required ranges of motion. The patient receives constant assistance, with the hand and wrist being attached to the end of the robotic arm and guided on a specific trajectory. The system is designed to support the weight of the patients upper limb, while performing exercises.

The proposed robotic device should provide a patient with five independent DOFs in the upper limb including, shoulder flexion and extension movement, shoulder abduction and adduction movement, shoulder rotation movement around its axis, elbow flexion and extension movement and shoulder rotation movement around its axis. The device needs to be designed to cover the workspace of a half-sphere with the radius of a patients arm length, centered at the shoulder of a patient. Unilateral training is required at a time.

The robotic device needs to be bidirectional, with enough number of DOFs and appropriate link structure in order to meet the requirements mentioned above. The requirements for a robotic rehabilitation device also include comfort, safety and low complexity.

\section{B. Mechanical Design}

The proposed system (Fig. 1) was designed as a stationary, end-effector based robotic device comprising of a basement A, six serial links E-J and a hand-holder plate N. The system design incorporates two curved links $\mathrm{H}$ and I. Link $\mathbf{J}$ and the 

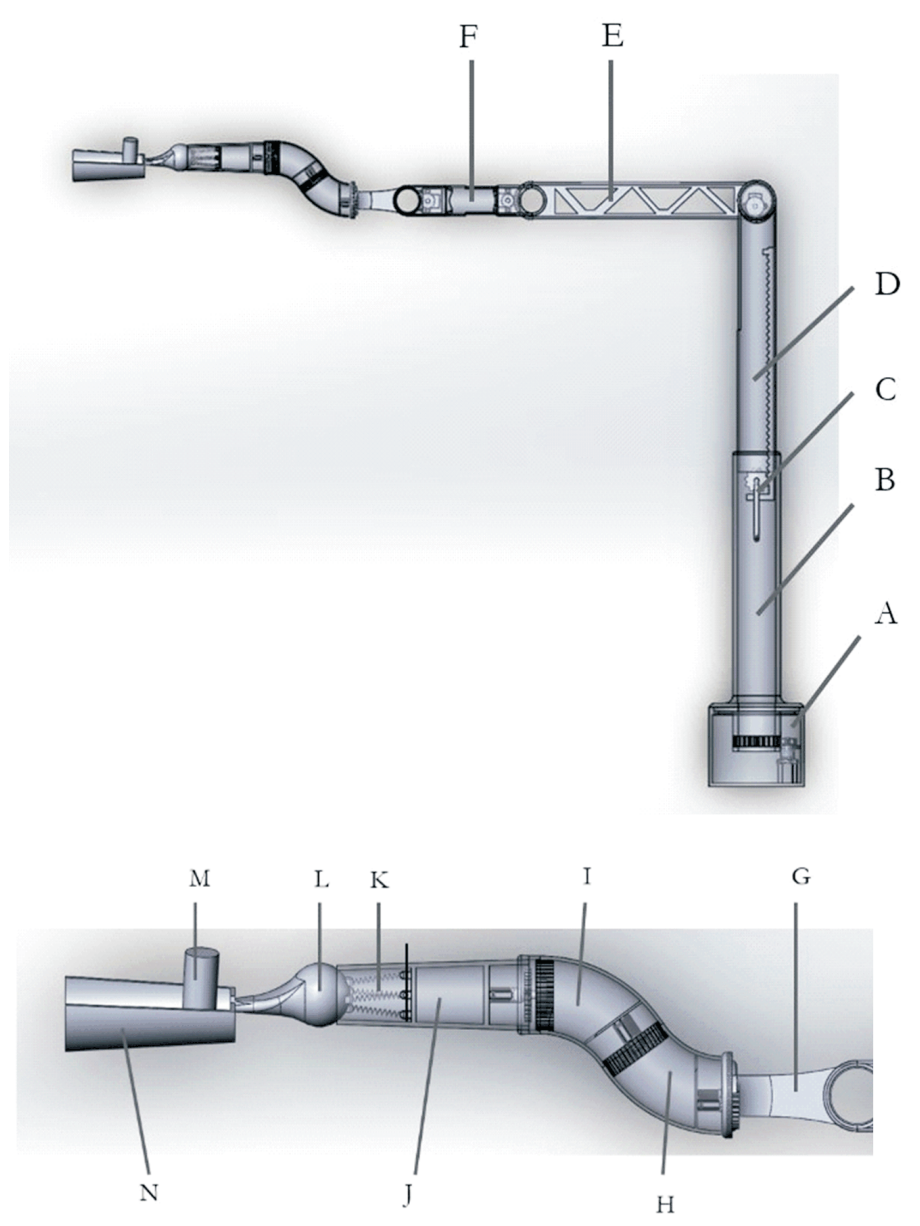

Fig. 1. Robotic exerciser design. Major components labeled: A - base for horizontal rotation, B - stationary base $\operatorname{link}(1), \mathrm{C}$ - height adjusting screw, $\mathrm{D}$ - base $\operatorname{link}(2)$ for vertical passive translation, $\mathrm{E}$ - translational $\operatorname{link}(1), \mathrm{F}$ translational $\operatorname{link}(2), \mathrm{G}$ - translational $\operatorname{link}(3), \mathrm{H}$ - rotational curved link(4), I - rotational curved $\operatorname{link}(5), \mathrm{J}$ - rotational $\operatorname{link}(6), \mathrm{K}$ - springs to hold the ball joint, $\mathrm{L}$ - passive ball joint, $\mathrm{M}$ - handle for the patients hand, $\mathrm{N}$ - hand-holder plate for the patients arm.

hand-holder plate $\mathrm{N}$ are connected through a ball joint $\mathrm{L}$. This ball joint is a passive joint, which is positioned by springs $\mathrm{K}$, that are are stiff and allow only little displacement of the ball joint axis in relation to the link $\mathbf{J}$ axis. Joints that interconnect the links are actuated by electromagnetic actuators and provide translational and rotational degrees of freedom (Fig. 2).

The patients hand and wrist part is fastened with elastic bandages to the hand-holder plate that can translate in a halfspherical workspace (Fig. 3). The shoulder and elbow joints of a patient are free and can move in compliance with his/her hand motion. The height of the robotic system is adjustable for patients of different height $\mathrm{H}$ (Fig. 2). In Fig. 2 baselink F can be pushed into and pulled out from the baselink $G$. At the desired position a height screw $\mathrm{C}$ in Fig. 1 fixes the structure. The whole structure, but the hand-holder plate, is to be made of aluminum; the hand-holder plate of carbon polymeric composites.

The robot was designed for use by patients with weight
A

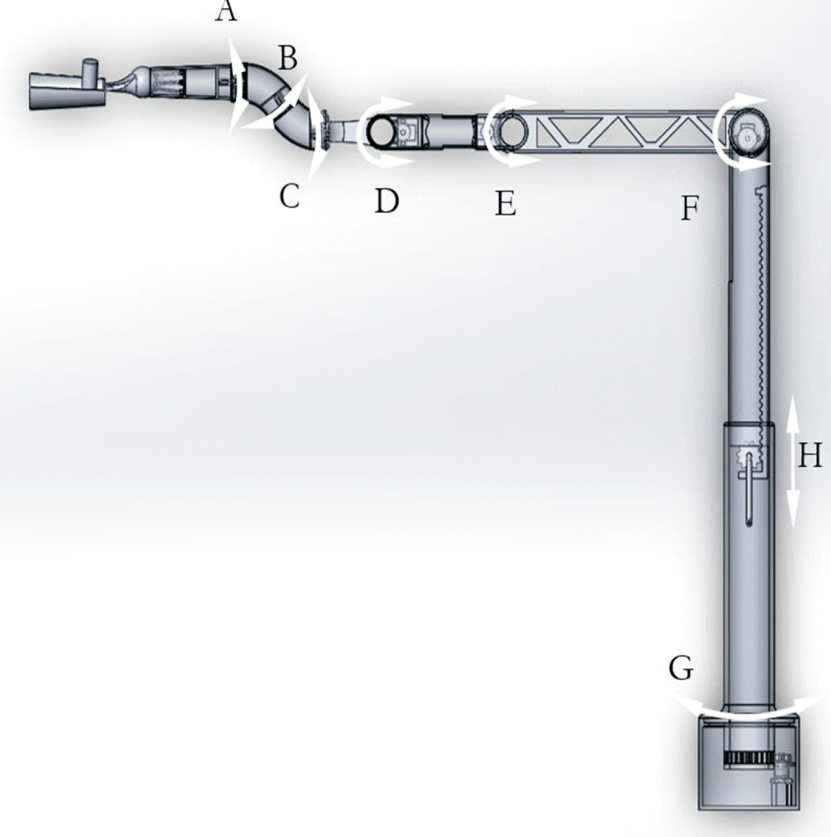

Fig. 2. Kinematic layout of the proposed robotic exerciser. (A) Actuated rotational joint(6). (B) Actuated rotational joint(5). (C) Actuated rotational joint(4). (D) Actuated translational joint(3). (E) Actuated translational joint(2). (F) Actuated translational joint(1). (G) Actuated rotational base joint. (H) Passive height-adjustable frame.

ranging from 50 to $120 \mathrm{~kg}$ and of the height between 160 and $190 \mathrm{~cm}$. According to calculations and data on properties of body segments, a human with $120 \mathrm{~kg}$ weight and $190 \mathrm{~cm}$ height has the upper limb of $6 \mathrm{Kg}$ in weight and $70 \mathrm{~cm}$ in length. Thus, the proposed manipulator was calculated using these assumptions. The 3D design of the robotic manipulator was made in the SolidWorks mechanical design software. The link dimensions of the robotic arm were selected as a result of optimization criteria. The optimization was based on factors like desired motion behavior and collision avoidance as well as the requirement of workspace. At first, in order to provide full extension and flexion to the patients arm, the robotic manipulator needs to be able to come into fully flexed and fully extended modes, respectively (Fig. 3a). Secondly, the length of the robotic arm should be long enough to provide full abduction-adduction to a patients arm (Fig. 3(b)). Moreover, mass compensation of the design mass was done by making holes in the link structures.

\section{Actuation}

The structure and operation of the manipulator depend on the type of an actuator used for its powering. Therefore, in the designing process it was important to select the most appropriate actuator type. Pneumatic, hydraulic and electromagnetic actuators have been considered as a possible powering source for the manipulator.

As the rehabilitation robot interacts with a human, the mechanism should be safe. Resiliency of the joints, or which 


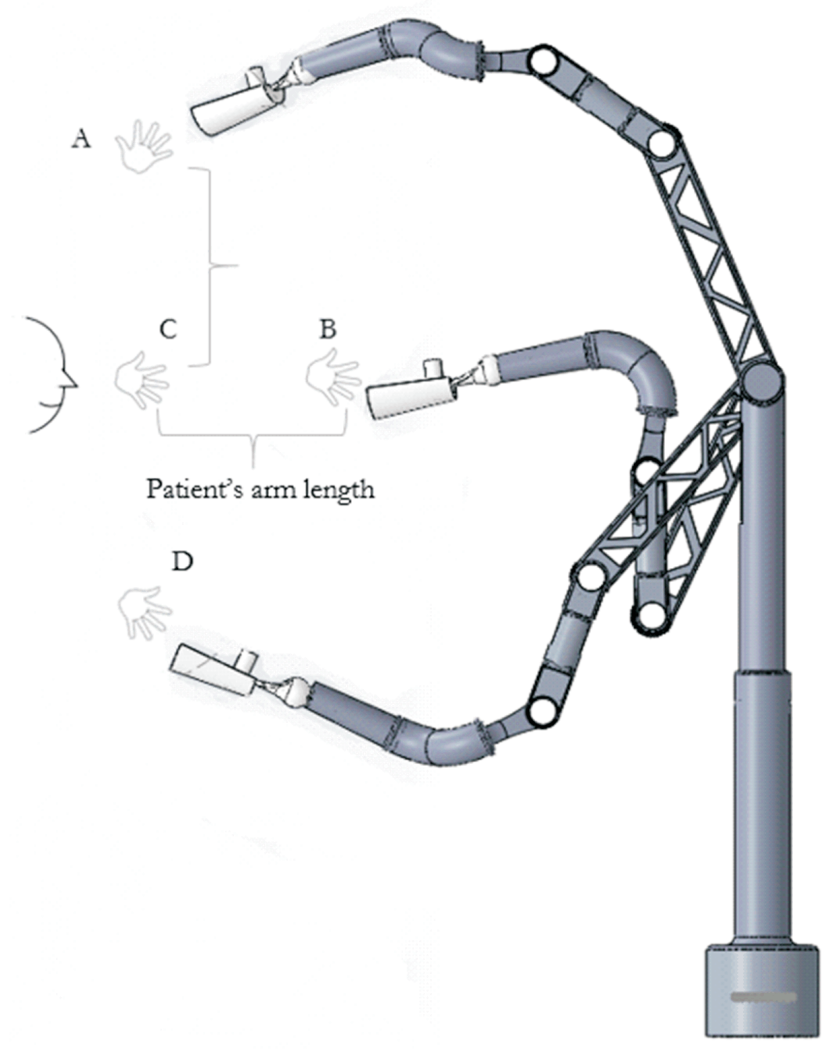

(a)

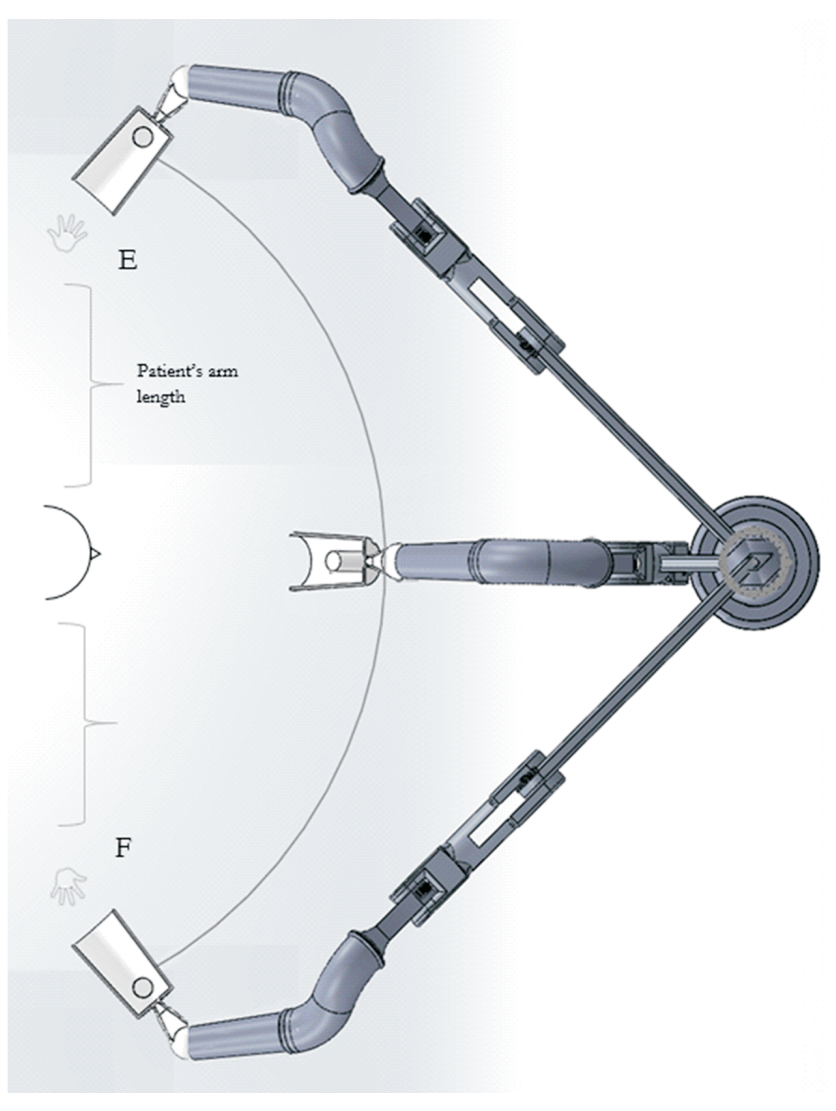

(b)

Fig. 3. Workspace of the robot. (a) Side View. (b) Top view. (A) and (D) Full shoulder extension-flexion. (B) and (C) Full elbow extension-flexion. (E) and (F) Full shoulder abduction-adduction

is called back-drivability, is a crucial characteristic for the safe robotic-arm operation around people. Back-drivable hydraulic/pneumatic actuators are either under development, or complicated. Therefore, electromagnetic actuators for the joint powering have been selected.

Selection of the joint motors is done according to torque level that is required to drive specified links or end effector. Fig. 4 shows the simplified representation of the six link robotic manipulator for beam deflection analysis. Here $M$ is the required torque for the translational joints D-F (Fig. 2) which needs to be calculated. The weight of the robotic arm $W$ is assumed to be uniformly distributed; whereas the weight of the patients upper limb $W_{h}$ is partly uniformly distributed, which resides on the hand-plate. The rest part of the arm which is free in space as a point mass $L$. Length $l$ and mass $m$ of the structure is considered to be from the attachment of the considered link - stationary point - to the end-effector.

The beam deflection analysis equations are derived as follows:

$$
\begin{gathered}
q(x)=-M(x-0)^{-2}+R(x-0)^{-1}-W(x-0)^{0}- \\
W_{\text {hand }}\left(x-\left(l-l_{\text {hand }}\right)\right)^{0}-L(x-1)^{-1} ; \\
V(x)=-M(x-0)^{-1}+R(x-0)^{0}-W(x-0)^{1}- \\
W_{\text {hand }}\left(x-\left(l-l_{\text {hand }}\right)\right)^{1}-L(x-1)^{0} ;
\end{gathered}
$$

$$
\begin{aligned}
M(x)= & -M(x-0)^{0}+R(x-0)^{1}-\frac{W}{2}(x-0)^{2}- \\
& \frac{W_{\text {hand }}}{2}\left(x-\left(l-l_{\text {hand }}\right)\right)^{2}-L(x-1)^{1} .
\end{aligned}
$$

Here, equations (1) and (2) represent loading and shear functions, respectively, equation (3) expresses a moment function.

Analysis of equations (1) - (3) at the end of the manipulator yields the following equations:

$$
\begin{aligned}
& V(l+)=R-W l-L-W_{h} l_{\text {hand }}=0 \\
& M(l+)=-M+R l-\frac{W}{2} l^{2}-\frac{W_{\text {hand }}}{2} l_{\text {hand }}^{2}=0 .
\end{aligned}
$$

Here, $M$ is the torque transmitted by the associated joint, $R$ is the reaction force at the joint, $W$ is the weight supported by the associated joint, $W_{\text {hand }}$ is the weight of the part of the patients arm that resides on the hand-holder plate, $l_{\text {hand }}$ is the length of the part of the patients arm that resides on the hand-holder plate and $l$ is the length of the robotic arm supported by the joint.

The required torques for the rotational joints A-C and base joint $\mathrm{G}$ (Fig. 2) are calculated in a different manner using the following equations, that provide rotational motion not in the 


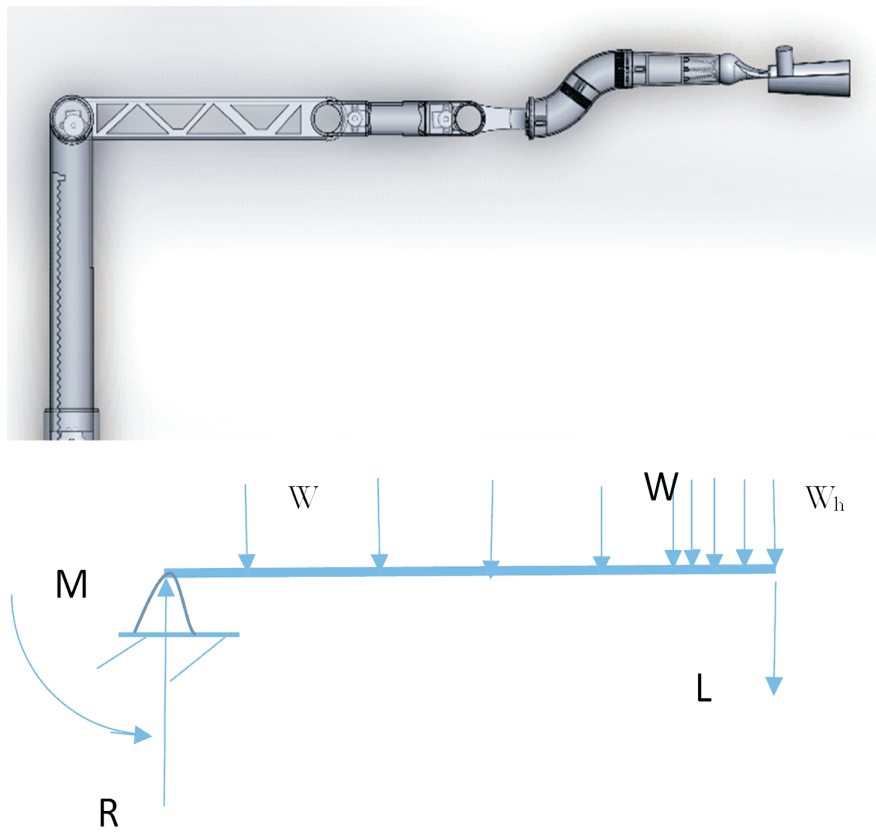

Fig. 4. Beam deflection analysis. Moment calculations.

vertical plane, but in the horizontal:

$$
\begin{aligned}
& T=I \alpha ; \\
& I=m r^{2},
\end{aligned}
$$

where $\alpha$ is the angular acceleration (assumed to be $\pi$ radians per second squared), $I$ is the moment of inertia, $m$ is the mass rotated by the joint, $r$ is the radius of rotation.

\section{RESUlts AND Discussion}

The electromagnetically actuated robotic manipulator design features a basement, two basement links, a height screw, six serial links interconnected by tree rotary joints in the vertical plane (Fig. 3) and four rotary joints in the horizontal plane, a passive ball joint (Fig. 3), springs and a hand-holder plate. The total length of the arm from the joint(1) to the end of the hand-holder is $140 \mathrm{~mm}$. The structure is to be produced of aluminum and carbon polymeric composites. Specifying the material type in the SolidWorks 3D CAD software the mass of the entire robotic system is calculated to be $5.84 \mathrm{~kg}$.

The robotic manipulator is fixed on the floor and a patient sits in the front of it. It is designed for the people with weight between 50 and $120 \mathrm{~kg}$ and height between 160 and $190 \mathrm{~cm}$. The patients hand enfolds the handle M (Fig. 3) on the handholder plate and follows the path specified by the end-effector. The robotic arm has 7 DOF and can exert bidirectional forces, while providing a patient with $3 \mathrm{DOF}$ in shoulder and $2 \mathrm{DOF}$ in elbow. The workspace of the robot is a half sphere in the front of a patient, centered at his exercised shoulder. The workspace has the radius of the patients arm length and the robotic manipulator is able to cover the workspace for the patient with arm length of $70 \mathrm{~cm}$ (Fig. 3).
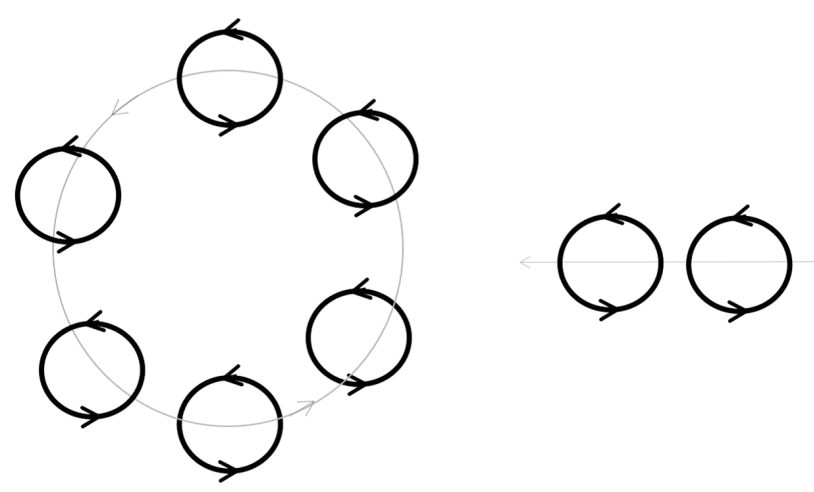

Fig. 5. Examples exercisers that could be performed by the robotic exerciser, because it has curved links in its design.

The novelty of the proposed rehabilitation robot for the upper limb is its ability to revolve the patients arm, while following the desired path. This is possible due to introducing curved links into the design. When joint $\mathrm{C}$ (Fig. 2) rotates link $\mathrm{H}$ (Fig. 3) and joint B (Fig. 2) does not rotate, link I (Fig. 3) revolves around the axis of joint C (Fig. 2) and, as a result revolves the hand-holder plate with the patients arm. At the same time, to not twist the patients wrist joint A (Fig. 2) rotates in the opposite direction of rotation of joint $\mathrm{C}$ (Fig. 2). Examples of the paths described above are on Fig. 5.

The robotic manipulator with all included equipment such as actuators, joints, fixed on the floor and balanced. The joints are actuated separately and, therefore, are independent in motion. High dexterity of the manipulator arm (Figs. 2 and 3) allows the robot to suit patients with different motor impairments. The robotic manipulator is able to provide a patient with complex movements of full arm extension-flexion, abductionadduction, as well as, operate in narrow, confined workspace area. Its ability to perform revolving motion of the arm, while conducting the exercises (Fig. 5), can be advantageous for the patients physical therapy. As a result, wider range of exercises makes the rehabilitation of the upper limb more effective.

The edges of the robotic structure are filleted and, therefore, will not injure the skin of the patient. The fixators are elastic and, therefore, will not interfere with the blood circulation of the patient. A flexible passive ball joint displaces when the wrist part of the patient arm to band and prevents the patients arm from feeling any discomfort.

\section{CONCLUSion}

In this paper, an end-effector based robotic manipulator for upper limb rehabilitation was developed. The purpose of which is to enhance the manual therapeutic training. It is provides a patient with 3 DOF in shoulder and 2 DOF in elbow, and is capable to manipulate a payload of $7 \mathrm{~kg}$. From the specifications mentioned above, the robotic manipulator meets all design requirements raised in Section II. It is powered by electromagnetic actuators, with justified pay load and dexterity. From the end-effector based rehabilitation robots available on market, proposed design for the robotic manipulator has 
particular advantages for the actuation and high range of workspace.

\section{REFERENCES}

[1] N. Bayona and et al., "The role of task-specific training in rehabilitation therapies," Topics in Stroke Rehabilitation, vol. 12, no. 3, pp. 58-65, 2005.

[2] R. Bonita and R. Beaglehole, "Recovery of motor function after stroke," Stroke, vol. 19, 1988.

[3] S. Cramer and J. Riley, "Neuroplasticity and brain repair after stroke." Current Opinion in Neurology, vol. 21, no. 1, pp. 76-82, 2008.

[4] B. D and N.HJ, "Cross-modal plasticity: where and how?" Nat. Rev. Neurosci, vol. 3, pp. 443-452, 2002.

[5] G. Kwakkel, B. Kollen, and R. Wagenaar, "Long term effects of intensity of upper and lower limb training after stroke: a randomised trial," Journal of Neurology Neurosurgery and Psychiatry, vol. 72, no. 4, pp. 473-479, 2002.

[6] G. Kwakkel and et al., "Intensity of leg and arm training after primary middle-cerebral-artery stroke: a randomised trial," Lancet, vol. 354, no. 9174, pp. 191-196, 1999.

[7] N. L. Holder and et al., "Cause, prevalence, and response to occupational musculoskeletal injuries reported by physical therapists and physical therapist assistants," Physical Therapy, vol. 79, no. 7, pp. 642-652, 1999.

[8] S. Hussain, S. Q. Xie, and G. Liu, "Robot assisted treadmill training: Mechanisms and training strategies," Medical Engineering \& Physics, vol. 33, no. 5, pp. 527-533, 2011.

[9] D. J. Reinkensmeyer, J. Emken, and S. Cramer, "Robotics, motor learning, and neurologic recovery," Annual Review of Biomedical Engineering, vol. 6, pp. 497-525, 2004.

[10] L. Kahn and et al., "Robot-assisted movement training for the strokeimpaired arm: Does it matter what the robot does?" Journal of Rehabilitation Research and Development, vol. 43, no. 5, pp. 619-629, 2006.

[11] L. Marchal-Crespo and D. Reinkensmeyer, "Review of control strategies for robotic movement training after neurologic injury," Journal of NeuroEngineering and Rehabilitation, vol. 6, no. 1, 2009.

[12] J. L. Emken and D. Reinkensmeyer, "Robot-enhanced motor learning: Accelerating internal model formation during locomotion by transient dynamic amplification," IEEE Transactions on Neural Systems and Rehabilitation Engineering, vol. 13, no. 1, pp. 33-39, 2005.
[13] R. J. Sanchez and et al., "Automating arm movement training following severe stroke: Functional exercises with quantitative feedback in a gravity-reduced environment," IEEE Transactions on Neural Systems and Rehabilitation Engineering, vol. 14, no. 3, pp. 378-389, 2006.

[14] R. Colombo and et al., "Robotic techniques for upper limb evaluation and rehabilitation of stroke patients," IEEE Transactions on Neural Systems and Rehabilitation Engineering, vol. 13, no. 3, pp. 311-324, 2005.

[15] S. E. Fasoli and et al., "Effects of robotic therapy on motor impairment and recovery in chronic stroke," Archives of Physical Medicine and Rehabilitation, vol. 84, no. 4, pp. 477-482, 2003.

[16] G. Kwakkel, B. J. Kollen, and H. Krebs, "Effects of robot-assisted therapy on upper limb recovery after stroke: A systematic review," Neurorehabilitation and Neural Repair, vol. 22, no. 2, pp. 111-121, 2008.

[17] P. Lum and et al., "Robot-assisted movement training compared with conventional therapy techniques for the rehabilitation of upper-limb motor function after stroke," Archives of Physical Medicine and Rehabilitation, vol. 83, no. 7, pp. 952-959, 2002.

[18] A. Stienenw and et al., "Design of a rotational hydroelastic actuator for a powered exoskeleton for upper limb rehabilitation," IEEE Transactions on Biomedical Engineering, vol. 57, no. 3, pp. 728-735, 2010.

[19] T. Sugar and et al., "Design and control of RUPERT: a device for robotic upper extremity repetitive therapy," IEEE Transactions on Neural Systems and Rehabilitation Engineering, vol. 15, no. 3, pp. 336-346, 2007.

[20] H. Lo and S. Xie, "Exoskeleton robots for upper-limb rehabilitation: state of the art and future prospects," Medical Engineering \& Physics, vol. 34, pp. 261-268, 2011.

[21] P. Lum and et al., "MIME robotic device for upper-limb neurorehabilitation in subacute stroke subjects: a follow-up study," Journal of Rehabilitation Research and Development, vol. 43, no. 5, pp. 631-642, 2006.

[22] L. Masia and et al., "Design and characterization of hand module for whole-arm rehabilitation following stroke," IEEE/ASME Transactions on Mechatronics, vol. 12, no. 4, pp. 399-407, 2007.

[23] H. Krebs and et al., "Robot-aided neurorehabilitation: a robot for wrist rehabilitation," IEEE Transactions on Neural Systems and Rehabilitation Engineering, vol. 15, pp. 327-335, 2007.

[24] D. J. Reinkensmeyer, J. Dewald, and W. Rymer, "Guidance-based quantification of arm impairment following brain injury: a pilot study," IEEE Transactions on Rehabilitation Engineering, vol. 7, pp. 1-11, 1999. 